\title{
Reducing medical errors in primary care using a pragmatic complex intervention
}

\begin{abstract}
This study aimed to develop an intervention to reduce medical errors and to determine if the intervention can reduce medical errors in public funded primary care clinics. A controlled interventional trial was conducted in 12 conveniently selected primary care clinics. Random samples of outpatient medical records were selected and reviewed by family physicians for documentation, diagnostic, and management errors at baseline and 3 months post intervention. The intervention package comprised educational training, structured process change, review methods, and patient education. A significant reduction was found in overall documentation error rates between intervention (Pre 98.3\% [CI 97.1-99.6]; Post 76.1\% [CI 68.1-84.1]) and control groups (Pre 97.4\% [CI 95.1-99.8]; Post 89.5\% [85.3-93.6]). Within the intervention group, overall management errors reduced from 54.0\% (CI 49.9-58.0) to $36.6 \%$ (CI 30.2-43.1) and medication error from 43.2\% (CI 39.2-47.1) to 25.2\% (CI 19.930.5). This low-cost intervention was useful to reduce medical errors in resource-constrained settings.
\end{abstract}

Keywords: Diagnostic errors; Medical errors; Medication errors; Nurse practitioners; Primary health care 